\title{
An Indoor Mobile Robot Localization in perspective of Analysis and Performance using Unscented Kalman Filter
}

\author{
Rashid Ali ${ }^{1,2, *}$, Yongping $\mathrm{He}^{1}$, Wenpeng $\mathrm{Fu}^{1}$ and Zhiqiang $\mathrm{Cao}^{1}$ \\ ${ }^{1}$ School of Information Engineering, Southwest University of Science and Technology, \\ Mianyang 621010, China \\ ${ }^{2}$ Department of Comupter Science, University of Turbat, Balochistan 92600, Pakistan
}

('Corresponding author's e-mail: alirashid@mails.swust.edu.cn)

Received: 8 November 2020, Revised: 19 May 2021, Accepted: 26 May 2021

\begin{abstract}
This paper describes a method in an indoor environment for the estimation and position, using an Unscented Kalman Filter (UKF). The UKF algorithm applied for the position estimation proposing a new measurement uncertainty model that fixes the error covariance according to the distance measurement. In addition, this approach sets the non-diagonal component of the error covariance matrix for the uncertainty of the speed information and the measurement uncertainty to a value other than zero. This method is evaluated through an experiment using a wheel-type mobile robot with an LRF sensor in an indoor environment. In this experiment, we differentiate the estimation execution of the proposed approach with a conventional method that does not employ an adaptive uncertainty model. Moreover, the results improved the estimation performance by setting the non-diagonal component of the error covariance to a value other than zero. The main emphasis of this paper is to implement a practical UKF method for location estimation of a mobile robot and analyze it with better performance.
\end{abstract}

Keywords: Position estimation, Robot localization, Unscented Kalman Filter (UKF), Error covariance matrix, Uncertainty measurement

\section{Introduction}

The filtering methods for state estimation are widely used in navigation and observation of a mobile robot. The typical filtering methods are the Extended Kalman Filter (EKF), Unscented Kalman Filter (UKF), and the particle filter (PF) methods [1]. This study estimates the position of the mobile robot by applying the unscented Kalman filter method among these filtering methods. The UKF can also be used in nonlinear systems and has the characteristics of particle filters that generate particles in a stochastic way. The number of particles increases in particle filters, so the calculation amount increases, and the processing speed gets slow. However, the UKF method uses a method of reducing the number of particles and weighing them using a deterministic approach. Therefore, it shows that the processing speed is relatively fast, and it is robust to the position estimation using the characteristics of the EKF [2].

The particles in the UKF method are expressed as sigma points. The UKF method can flexibly improve the performance owing to many adjustable design variables. However, if the design variables are not properly set, the estimation becomes unstable [3]. In general, in the case of a system using a UKF method, the estimation result is greatly influenced by the size of the error covariance and generating the sigma point. Another important thing is that multiple sigma points should not be concentrated in one place and not be too scattered; so that the sigma point does not deviate from the boundary on the map. Many types of research on the UKF method are in progress, such as comparing different filtering methods and sensor fusion [4]. The UKF is presently used in a wide range of applications, from target tracking [31]. Some other types of data fusion studies aimed at improving the performance of mobile robots [32]. In the filtering methods for estimation, the sensor's accuracy has an important influence on the estimation result when the position is corrected with the information of the LRF sensor.

When applying the algorithm, the uncertainty value for the sensor information should be considered appropriately. The wheelchair position estimation issues in indoor scenarios with state vector fusion (SVF) and measurement fusion (MF) approaches in nonlinear systems where the 2-odometer is positioned on the axes of the wheels with a magnetometer to estimate location and orientation [33]. In the previous 
study, the uncertainty value of the measured sensor is expressed as one fixed value [5]. However, in the part where the measured sensor distance is short, there is a little noise, and in the part where the sensor distance is measured long, there is a lot of noise. Therefore, the uncertainty of the measured sensor information must be considered differently. Moreover, this study designs an equation to measure sensor information and an uncertainty value proportional to the sensor distance [6,7]. This equation reduces the error rate between the measured position and the estimated position and improves the estimation result.

In $[8,9]$, the location is known using the measured laser sensor information when using the estimation algorithm. Considering the uncertainty of the sensor, most studies make the non-diagonal components of the error covariance matrix zero. Suppose the calculated sensor distance corresponding to one calculated sensor distance from the predicted robot position is different. In that case, the remaining measured sensor distance and the calculated sensor distance are also different. Therefore, through the experiment of giving non-diagonal components of the error covariance matrix to a non-zero value, an appropriate value was found and implemented according to the actual system. Applying a non-zero value reduces the estimation error rate and improves the estimation performance. Therefore, this paper differentiates the estimation execution of the proposed approach by employing an adaptive uncertainty model. The results increased the estimation performance by adjusting the non-diagonal component of the error covariance to a value other than zero. The major goal of this study is to develop and test a realistic UKF approach for mobile robot position estimation for better performance.

The subsequent sections of this paper are organized as follows: The UKF algorithm section represents the initialization of the state variables, applications of motion, and sensor models in detail. The adaptive sensor uncertainty error covariance design and non-diagonal component design of error covariance matrix sections are concisely summarized. The simulation results and discussion section thoroughly presents and describes the control parameter, the experiment of sensor design uncertainty, and non-diagonal component comparison. The last section concludes the paper with future scope.

\section{Unscented Kalman Filter algorithm}

In general, the method used when applying the UKF, i.e., Unscented Transform (UT) method and Scaled Unscented Transform (SUT) method [10]. Also, there are the Cholesky decomposition method and Matrix square root method for generating sigma points. In this research, the UT method and Cholesky factorization are applied to the algorithm.

The Kalman UKF filter is used for the estimation of states in nonlinear systems. Although it is advantageous, in case care has been taken when entering the variables, the filter may not converge if incorrect measurements are entered. It occurs, for example, when the noise disturbing the system does not have a uniform density function.

\section{State variable initialization}

The state variable to be estimated in this research is $\mu_{t}=\left(x_{t}, y_{t}, \theta_{t}\right)^{T}$, which indicates the localization of the mobile robot. Moreover, $\mu_{t-1}^{a}$ represents the average value of the state variable having a Gaussian distribution with a mean of zero for the localization information $t-1$ at $\mu^{t}$ and the uncertainty for the internal and external sensors. As shown in Table 1 are initialized by $M_{t}$ and $Q_{t}$ for the uncertainty of the sensor information, which indicates the state of the sensor noise [11]. Furthermore, the uncertainty of the sensor information is continuously used when performing the algorithm process. Finally, the error covariance for the robot position is represented and initialized by $\Sigma_{t-1}[12]$.

Table 1 Parameters used in UKF.

\begin{tabular}{cc}
\hline Parameters & Description \\
\hline$\mu_{t-1}^{a}$ & Augmented state mean \\
$\Sigma_{t-1}$ & Augmented position error covariance \\
$M_{t}$ & Uncertain error covariance locomotion \\
$Q_{t}$ & Uncertain error covariance measurement \\
\hline
\end{tabular}


The Unscented transformation is a concept similar to Monte Carlo simulation, but it is possible to select and use the weights of each sample so that it is not suitable for real-time calculation due to a large amount of calculation in Monte Carlo simulation.

In Figure 1, the Unscented Kalman filter (UKF) is the origin of Unscented transformation (UT), a technique to calculate the state variable facts of a nonlinear transformation. A discrete variant of the system with additional white Gaussian noises has been defined the $L$ dimension state variable $\mu$ with mean $\bar{\mu}$ and $\Sigma_{t}$ uncertain error covariance is approximate by $2 L+1$ where the weighted sigma points is used in this paper among many UKF approaches specified in Eqs. (1) and (2).

$$
\left\{\begin{array}{l}
\mu^{0}=\bar{\mu} \\
\mu^{i}=\bar{\mu}+\left(\sqrt{(L+t) \Sigma_{t}}\right)_{i}, i=1, \ldots, L \\
\mu^{i}=\bar{\mu}+\left(\sqrt{(L+t) \Sigma_{t}}\right)_{i-L}, i=L+1, \ldots, 2 L
\end{array}\right.
$$

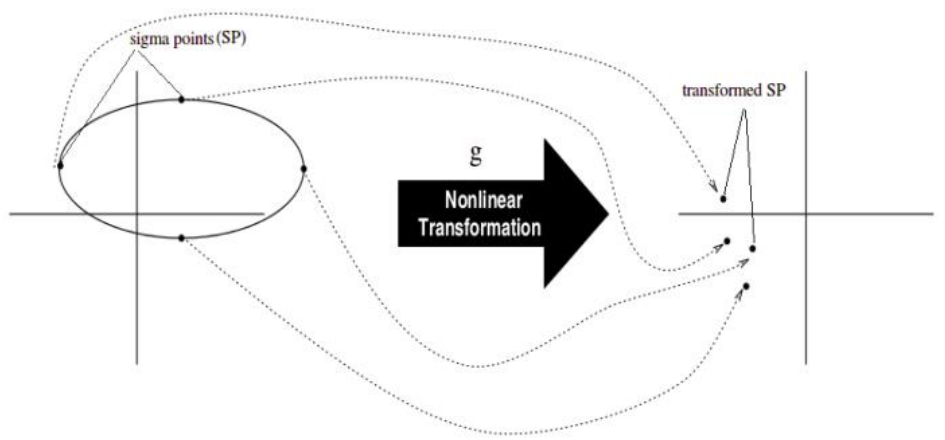

(a)

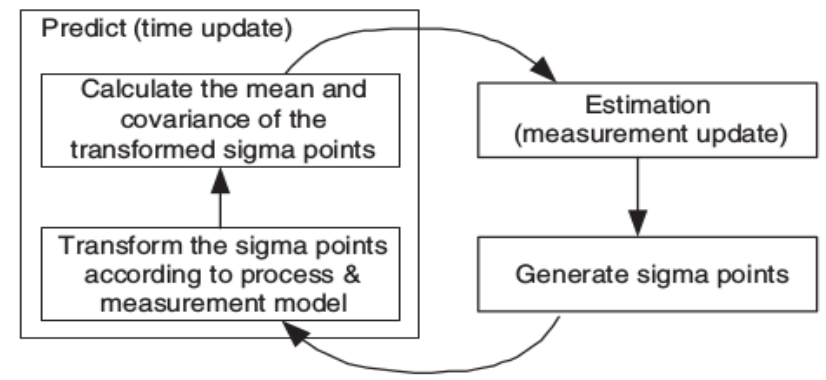

(b)

Figure 1 (a) Unscented transformation principle, and (b) An Unscented Kalman Filter process.

The weight $w^{i}$ is calculated using Eq. (2).

$$
\left\{\begin{array}{l}
w_{0}^{m}=t /(L+t) \\
w_{0}^{c}=t /(L+t)+\left(a-a^{2}+\beta\right) \\
w_{i}^{c}=w_{i}^{m}=1 /[2(t+L)], i=1, \ldots, 2 L
\end{array}\right.
$$

Where, the scaling parameter represented as $t=\alpha^{2}(L+\gamma)-L$, the propagated sigma points expressed as $\gamma$ around $\bar{\mu}$ setting as non-zero value, where $\gamma$ considered as secondary scaling parameter to set it zero normally, and $\beta$ is a parameter for integrating some prior knowledge of $\mu$ distribution. The matrix square root of $\left.(L+t) \Sigma_{t}\right)$ from $\left(\sqrt{\left.(L+t) \Sigma_{t}\right)}\right.$, where the $i^{\text {th }}$ represented the row or column and $w_{i}$ is the sigma points weight linked with $i^{\text {th }}$ point. 


\section{Motion model application}

The UKF algorithm uses sigma points to estimate nonlinear state transformation [13]. Sigma points can be predicted the position of the robot and calculate the Eq. (3) to indicate the state of the sigma points.

$x_{t-1}^{a}=\left(\mu_{t-1}^{a}+\gamma \sqrt{\sum_{t-1}^{a}} \mu_{t-1}^{a}-\gamma \sqrt{\sum_{t-1}^{a}}\right.$

In Eq. (3), $\gamma$ is a design variable used for the calculation of the scattered sigma points and $\sqrt{\sum_{t-1}^{a}}$ is obtained by Cholesky decomposition. The $\Sigma_{t-1}^{a}$ is the error covariance for robot position and $\Sigma_{t-1}$ is the uncertainty of the sensor information [14], where the size of the sigma point matrix is $24 \times 24$. Eq. (4) applies in the motion model using the sigma point obtained from Eq. (3).

$\bar{X}_{t}^{x}=g\left(u_{t}+x_{t}^{u}, x_{t-1}^{x}\right)$

Eq. (4) performs the dead reckoning of $x, y, \theta$ to find the positions of 49 sigma points, while the $g$ expressed the nonlinear transition state function, and the Control $u_{t}$ is used for the sampling of new robot pose according to the motion model for each state accordingly. Eqs. (5) and (6) calculate the expected average value $\bar{\mu}_{t}$ and the error covariance $\bar{\Sigma}_{t}$ of the predicted position of the robot using the results and weights of the estimated position.

$$
\begin{aligned}
& \bar{\mu}_{t}=\sum_{i=0}^{2 L} w_{i}^{(m)} \bar{x}_{i, t}^{x} \\
& \bar{\Sigma}_{t}=\sum_{i=0}^{2 L} w_{c}^{(m)}\left(\bar{x}_{i, t}^{x}-\bar{\mu}_{t}\right)\left(\bar{x}_{i, t}^{x}-\bar{\mu}_{t}\right)^{T}
\end{aligned}
$$

In Eq. (3) and $w_{i}, w_{c}$ represent the weights, $x_{t-1}^{a}$ are the sigma points which described the previous states and $\bar{x}_{i, t}^{x}$ are measurement noise, respectively. In Eqs. (5) and (6), which are obtained by an unscented transform method [15]. The state variables for the unscented conversion represent as $x, y, \theta$, straight speed, rotation speed, and 19 sensor distance information.

\section{Sensor model application}

The 2D sensor SICK LMS511 Laser Scanner ver.2014 from SICK in Figure 2 is used to correct the predicted position.

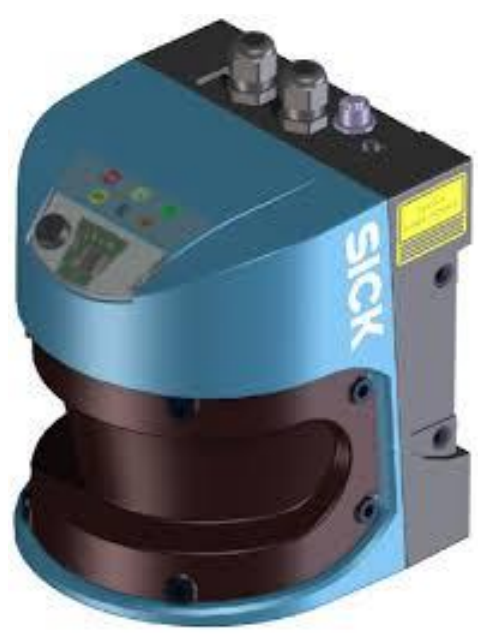

Figure 2 Laser Range Finder for distance measuring. 
In [16], the laser distance measuring sensor detects the interface using 19 sensor distances each with $10^{\circ}$ intervals from $0^{\circ}$ to $190^{\circ}$ on the right shown in Figure 2. The error covariance of the sensor $Q_{t}$ has a matrix of size $19 \times 19$, considering the uncertainty of 19 sensor distances, as shown in Eq. (7);

$Q_{t}=\left[\begin{array}{ccc}\sigma_{1}^{2} & \ldots & 0 \\ \vdots & \ddots & \vdots \\ 0 & \cdots & \sigma_{19}^{2}\end{array}\right]$

To calculate the distance of 19 sensors, each with a distance of $10^{\circ}$ from the 24 sigma point positions to the boundary line is shown in Eq. (8).

$$
\bar{Z}_{t}=h\left(\bar{x}_{t}^{x}\right)+x_{t}^{z}
$$

As the $x_{t}^{z}$ is an error for $x, y$, and $\theta$ measured in the laser sensor by using the sigma point from Eq. (8), the mean $\hat{x}_{t}$ and error covariance $S_{t}$ are obtained using Eqs. (9) and (10).

The sensor distances obtained by calculating the weight of the sigma point as given in Eq. (8) where $h$ represents the measurement transition function. In Eq. (9), the average value of the sensor distance weight is obtained with mean and covariance for $\hat{x}_{t}$ using the weighted sample mean and covariance of the posterior sigma points. Moreover, in Eq. (10), the error covariance is obtained for the sensor distance [17].

$$
\begin{aligned}
& \bar{x}_{t}=\sum_{i=0}^{2 L} w_{i}^{(m)} \bar{x}_{i, t}^{x} \\
& S_{t}=\sum_{i=0}^{2 L} w_{i}^{(c)}\left(\bar{Z}_{i, t}-\hat{x}_{t}\right)\left(\bar{Z}_{i, t}-\hat{x}_{t}\right)^{T}
\end{aligned}
$$

Eq. (11) calculates the cross-covariance using the state variable to be estimated and the calculated sensor distance. The Kalman gain is obtained using Eq. (12).

$$
\begin{aligned}
& \sum_{t}^{x, t}=\sum_{i=0}^{2 L} w_{i}^{(c)}\left(\bar{x}_{i, t}^{x}-\bar{\mu}_{t}\right)\left(\bar{Z}_{i, t}-\hat{z}_{t}\right)^{T} \\
& S_{t}=\Sigma_{t}^{x, z} S_{t}^{-1}
\end{aligned}
$$

The difference between the actual measured sensor distance and the sensor distance is multiplied by the Kalman gain in Eq. (9), and added to the predicted state variable $\bar{\mu}_{t}$ to finally calculate the estimated position as shown in Eqs. (13) and (14) [18].

$$
\begin{aligned}
& \mu_{t}=\bar{\mu}_{t}+K_{t}\left(z_{t}-\hat{z}_{t}\right) \\
& \Sigma_{t}=\bar{\Sigma}_{t}-K_{t} S_{t} K_{t}^{T}
\end{aligned}
$$

\section{Adaptive sensor uncertainty error covariance design}

Once the system mechanism and measurement noise statistics are understood prior, the adaptive sensor methods can provide accurate estimates of the system. At hand, most practical applications in analytical terms, these statistics can't be understood because they are closely related to the form of the system where the complexities of the sensor motion are complicated in our cases. In such cases, the use of the statistics given will degrade system efficiency. The rapid reflection of variations in the noise statistics from internal factors is needed to improve the estimation accuracy. 
The $Q_{t}$, which has uncertainty about 19 sensor distances, was designed to adaptively change with respect to sensor distances instead of being determined as a single value [19]. The expected effect is to improve the estimation performance by making the measurement noise for the LRF sensor information proportional to the actual distance information. The method of adaptively changing the measurement uncertainty value is expressed in Eq. (15).

$Q_{t, i}=\sigma_{r, i}^{2}+\gamma_{s} w_{s, i},(i=1, \ldots, 19)$

$\sigma_{r}^{2}$ uses the parameter value which is suitable for the system model in Eq. (7), and $w_{c}$ is obtained by using Eq. (17) for the weights of the 19 sensor distances at $t-1$.

$$
\begin{aligned}
& z_{\text {total }}=\sum_{i=1}^{19} z_{t-i, i}(i=1, \ldots, 19) \\
& w_{s, i}=z_{t-1, i} / z_{\text {total }}(i=1, \ldots, 19)
\end{aligned}
$$

In Eq. (15), $\gamma_{s}$ is a parameter that controls the uncertainty value of the sensor information and has a constant value that can determine the weight for each sensor distance.

\section{Non-diagonal component design of error covariance matrix}

The non-diagonal components of the error covariance matrix in position estimation have a significance influence on the estimation performance [20]. The part to consider the non-diagonal components of the error covariance matrix is $\Sigma_{t-1}, M_{t}, Q_{t}$.

First, the initial error covariance $\Sigma_{t-1}$ at $t-1$, makes the non-diagonal components zero. In order to represent the sigma points, in $\sqrt{\sum_{t-1}^{a}}$ performs the Cholesky decomposition method. At this time, if the non-diagonal components of the initial error covariance $\Sigma_{t-1}$ are not zero, then Cholesky decomposition is impossible because the lower triangular component is not positive definite [21]. Therefore, the nondiagonal components of the initial error covariance $\Sigma_{t-1}$ are determined to be zero. The error covariance calculated at $\Sigma_{t}$ becomes a positive definite matrix at the next time $t-1$, so that the Cholesky decomposition is possible. The $M_{t}$ represents the uncertainty of the sensor information as given in Eq. (18);

$$
M_{t}=\left[\begin{array}{cc}
\sigma_{1} v_{t}^{2}+\sigma_{2} w_{t}^{2} & 0.0001 \\
0.0001 & \sigma_{3} v_{1}^{2}+\sigma_{4} w_{t}^{2}
\end{array}\right]
$$

If the values of the non-diagonal components of the error covariance matrix are small [22], the linear velocity $M_{t}$ and the rotational speed $v_{t}^{2}$ in the velocity information $w_{t}^{2}$ have less effect on each other. The non-diagonal components for $Q_{t}$ are similar to Eq. (19) by adding the non-diagonal components in Eqs. (7), (18) and (19) are used at constant values until the estimation is completed.

$Q_{t}=\left[\begin{array}{ccc}\sigma_{1}^{2} & \ldots & 0.0001 \\ \vdots & \ddots & \vdots \\ 0.0001 & \ldots & \sigma_{19}^{2}\end{array}\right]$

Eqs. (18) and (19) are used at constant values until the estimation is completed.

\section{Simulation results and discussion}

The simulation has been performed based on UKF and UT, i.e., sigma points to the analysis and performance of the robot position estimation. As in the experiment, map information obtained from the location in Figure 3 and encoder information from the NRLAB02 robot is obtained and used to track the 
sensor data. In addition, the sensor data obtained by LMS511 ver.2014 of SICK where the accuracy of LMS511 is $10 \mathrm{~mm}^{\circ}$. The program for the estimation experiment LINUX 14.04 GCC with the system specification (processor Intel ${ }^{\circledR}$ Core $^{\mathrm{TM}}$ i7-4510U CPU @ $2.00 \mathrm{GHz} \times 4$, RAM 8GB and OS 64bit) has been used. The map shown in the experiment is based on a grid map, and the minimum distance between the grids is $9 \mathrm{~mm}^{\circ}$. The green line indicates the mileage is about $54 \mathrm{~m} \times 10 \mathrm{~m}^{\circ}$.

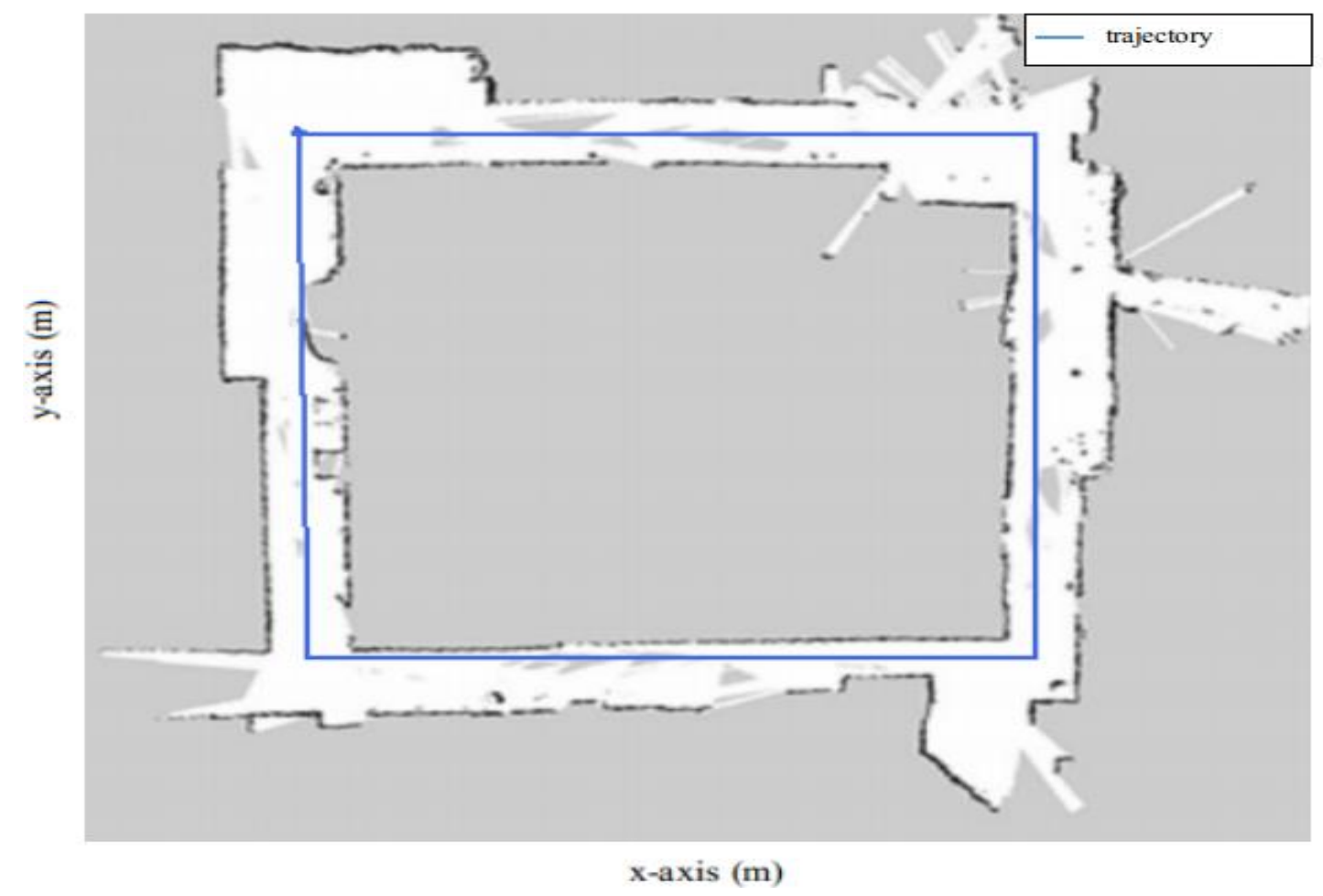

Figure 3 Map environment and robot driving route.

\section{Control parameter}

To apply UKF, the algorithm is applied in this experiment using the UT method and Cholesky decomposition method [23]. The important thing is that if you don't use the adjustable parameter values properly, it will decrease the estimation performance. Accordingly, the adjustable parameter values used for position estimation are summarized, as shown in Table 2.

Table 2 Control parameter with proposed value.

\begin{tabular}{cc}
\hline Parameters & Proposed value \\
\hline$\alpha_{1}$ & 0.0300 \\
$\alpha_{2}$ & 0.0300 \\
$\alpha_{3}$ & 0.0300 \\
$\alpha_{4}$ & 0.0300 \\
$\sigma_{1}^{2}, i=1, \ldots .19$ & $0.9^{2}$ \\
$\alpha_{1} \ldots \alpha_{4}$ & $0.1^{2}$ \\
$\gamma_{s}$ & 0.05 \\
$\gamma$ & 2.1268 \\
\hline
\end{tabular}


$\alpha_{1} \ldots \alpha_{4}$, where $\sigma_{1}^{2}, i=1, \ldots, 19$ represents the noise of the sensor information. $\Sigma_{t-1}$ represents the initial parameter of the error covariance [24], and $\gamma_{s}$ represents the weight to adjust the measurement noise of the sensor information. $\gamma$ represents a parameter that can control the degree of dispersion of sigma points when applying the Cholesky decomposition method.

\section{Sensor design uncertainty comparison experiment}

TThe first experiment compares the estimation method using the method of determining the uncertainty value of the existing measurement sensor as one and the estimation method by changing the uncertainty value of the measurement sensor using the sensor distance that changes every hour as a weight. The UKF method on RMSEs for robot position estimates is evaluated using the same velocity and noise parameters. The method to verify the distance error of the estimated robot position is the average $\mathrm{E}$ (error), standard deviation $\sigma$ (error), maximum error distance (max.), Standard error of the distance difference between the estimated position and the driving trajectory Root Mean Squares Error (RMSE) $[25,26]$. The driving trajectory means the blue line in Figure 3, which the robot moves relative to this line.

Table 3 showed 2 verification results when the estimation was completed. Figure 4 shows the experimental results when the measurement noise of the sensor information is one, and Figure $\mathbf{5}$ shows the experimental results of adaptively changing the measurement noise using the proposed method. In addition, the distance information of the measurement sensor indicates a distance compared to the existing measurement distance when the robot measures the navigation path. Therefore, if the parameter is determined incorrectly in this condition, the sigma point deviates from the boundary line. The estimated distance is reduced because the sensor distance calculated by the algorithm differs from the measured sensor distance [27].

The experiment varied the uncertainty value in proportion to the size of the external sensor data, and Changed uncertainty values were used by applying the weights. When measuring the distance of the LRF sensor, it deviates from the boundary line on the map and doubles the measurement distance. And the noise is loud in the part that is measured long in the center. Therefore, there is a massive difference between the sensor distance value calculated by the algorithm and the measured sensor distance in a specific section. The position is estimated toward the short measured side [28]. At this time, if the sigma point deviates from the boundary, the estimation is not made completely. Therefore, it is possible to reduce the estimation error by setting the uncertainty value of the adaptively changing measurement sensor.

Table 3 Comparison of measurement uncertainty models experimental results.

\begin{tabular}{ccc}
\hline Symbol usage & $\begin{array}{c}\text { measurement uncertainty } \\
\text { (Fixed) }\end{array}$ & $\begin{array}{c}\text { measurement uncertainty } \\
\text { (Adaptive) }\end{array}$ \\
\hline as an Error & 0.2803 & 0.1923 \\
$\sigma$ as an Error & 0.1834 & 0.0901 \\
Max. (Maximum) & 0.7857 & 0.8102 \\
RMSE (Root Mean Square Error) & 0.2989 & 0.3010 \\
\hline
\end{tabular}




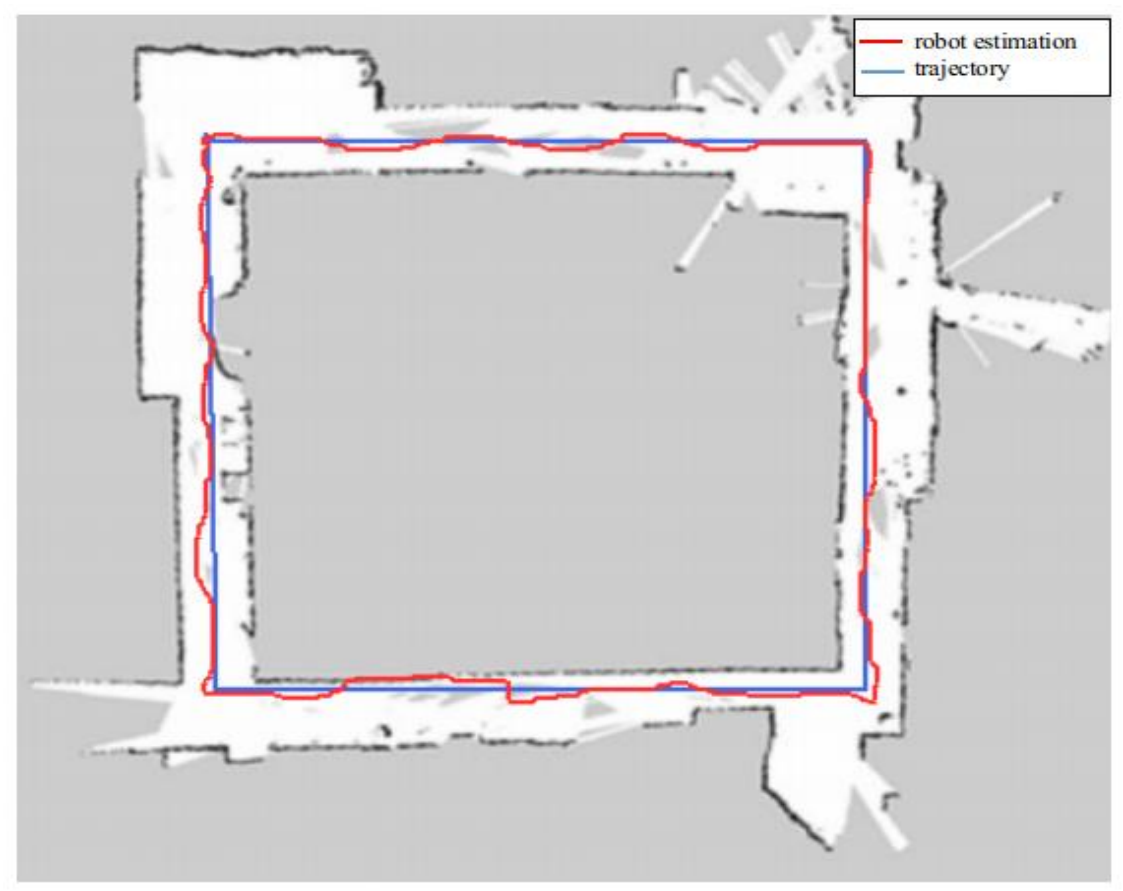

(a)

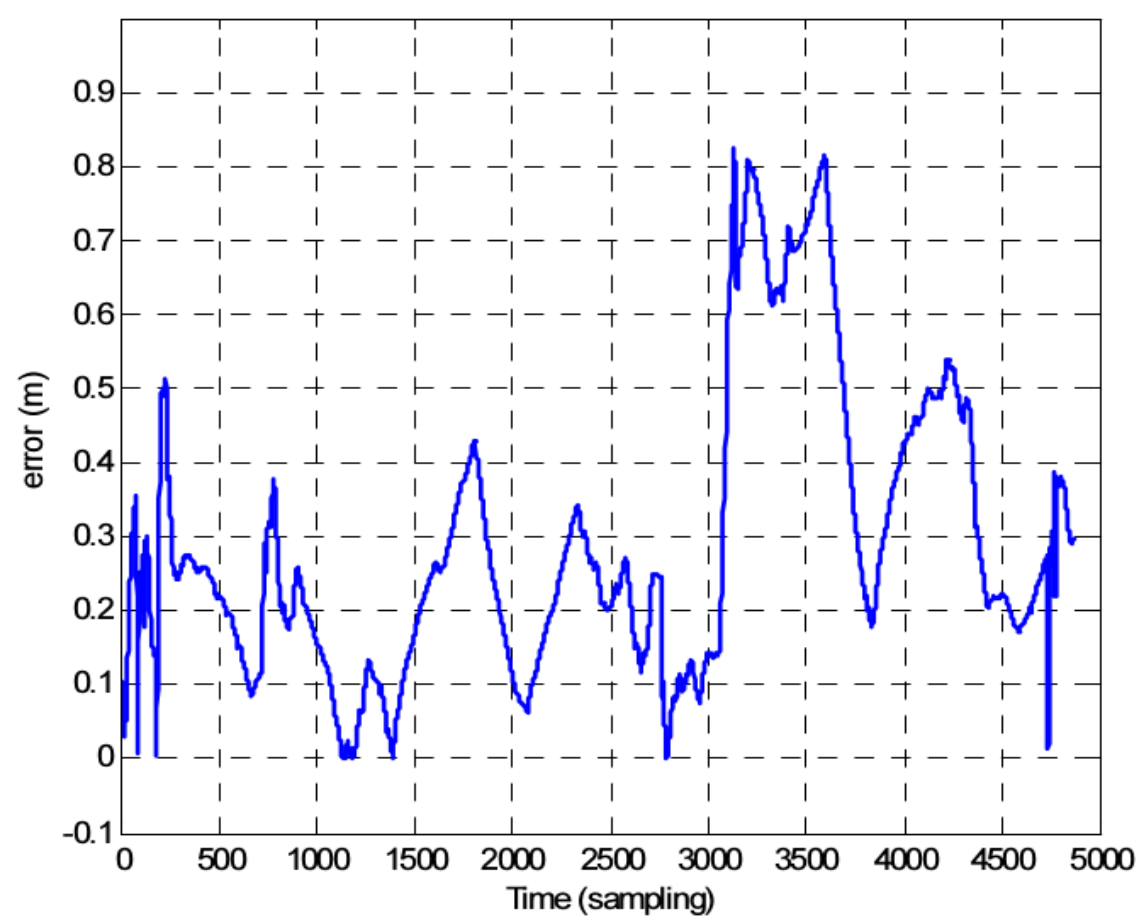

(b)

Figure 4 Results (a) fixed measurement uncertainty model, and (b) unscented transform and Cholesky decomposition errors. 


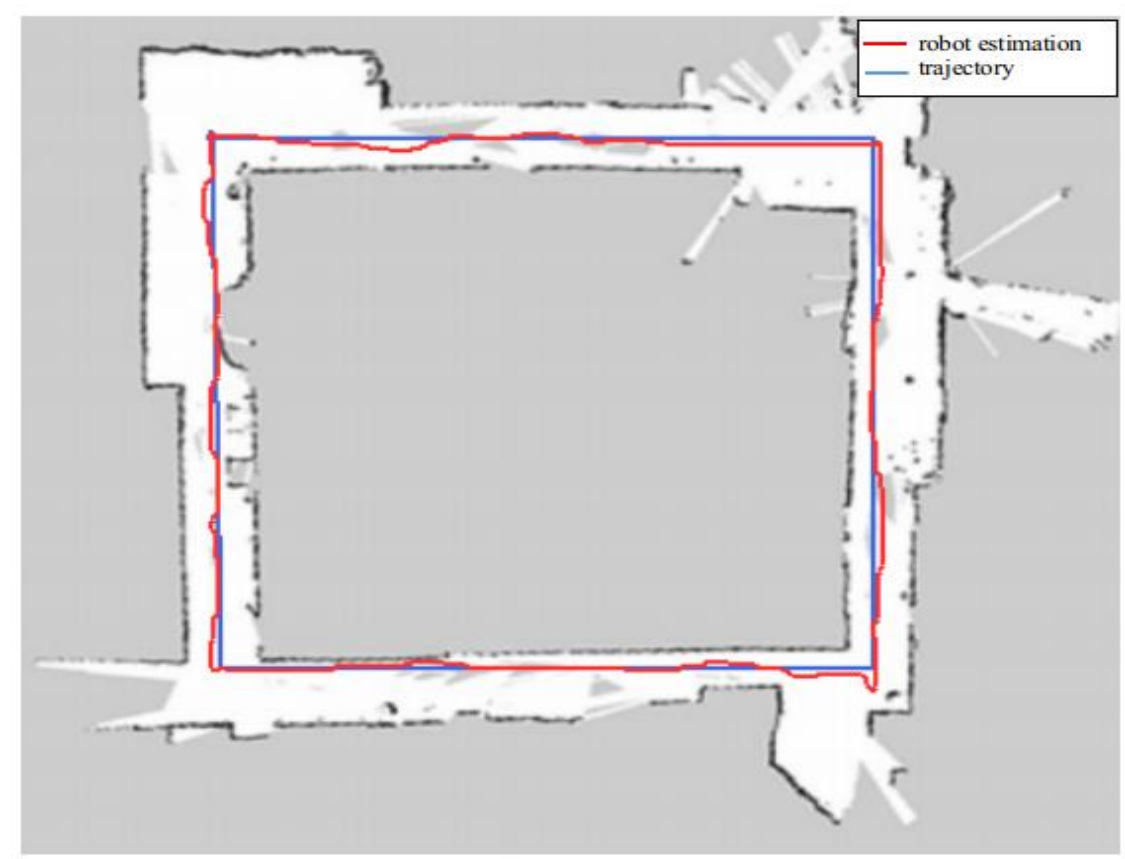

(a)

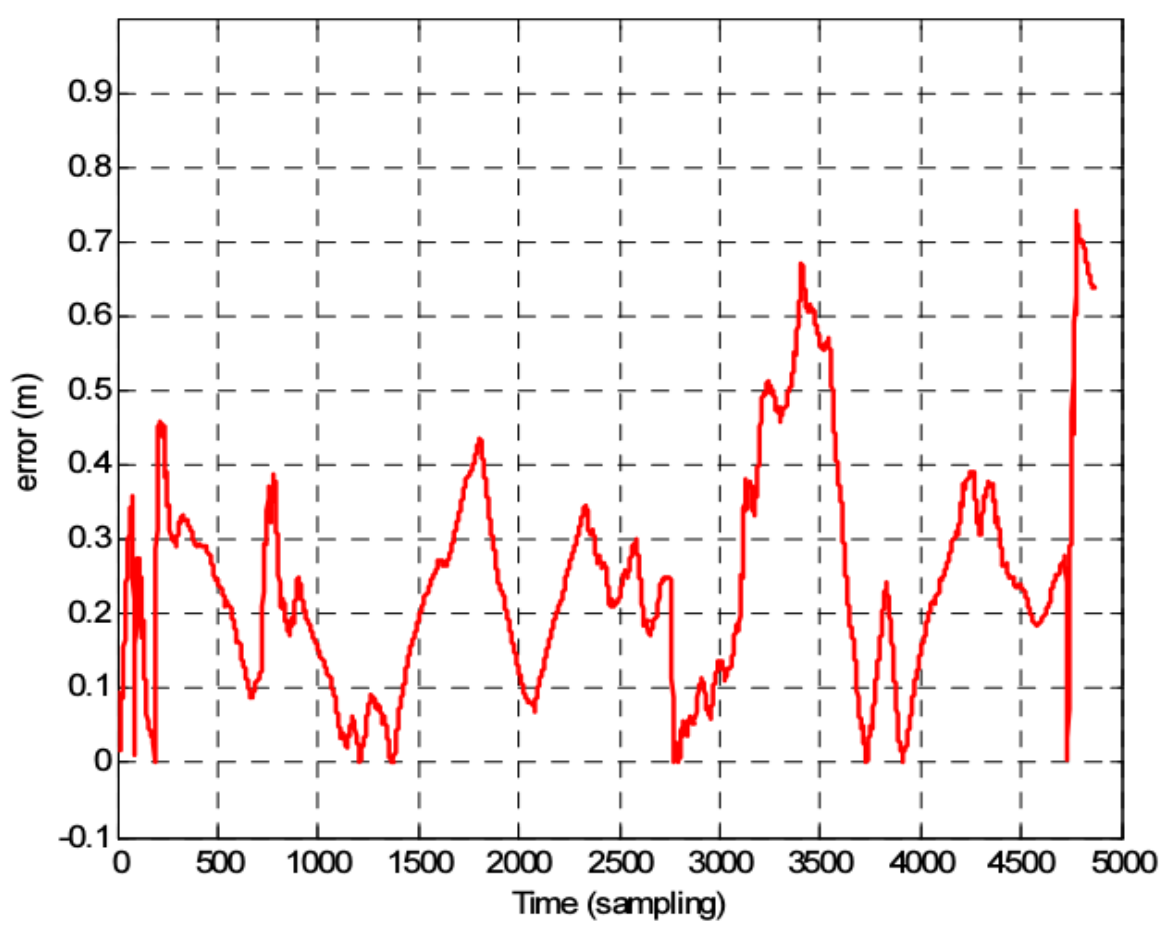

(b)

Figure 5 Results (a) an adaptive measurement uncertainty model, and (b) unscented transform and Cholesky decomposition errors. 


\section{Non-diagonal component comparison experiment}

The following experiment compares the estimated change according to the value of the nondiagonal component of the error covariance matrix. The estimation experiments have been observed when the non-diagonal component values of the error covariance matrix have been zero when non-zero constant values were given [29]. Table 4 shows the verification results obtained from the 2 estimation results when the diagonal ratio component of the error covariance matrix is zero in case the value is present.

Table 4 Non-diagonal components effects of the error covariance matrix.

\begin{tabular}{lcc}
\hline Symbol Usage & $\begin{array}{c}\text { measurement uncertainty } \\
\text { (Fixed) }\end{array}$ & $\begin{array}{c}\text { measurement uncertainty } \\
\text { (Adaptive) }\end{array}$ \\
\hline$E$ as an Error & 0.1912 & 0.1831 \\
$\sigma$ as an Error & 0.1790 & 0.1610 \\
Max. as Maximum & 0.7972 & 0.6982 \\
RMSE (Root Mean Square Error) & 0.3183 & 0.3010 \\
\hline
\end{tabular}

If a value is given to the non-diagonal component of the initial error covariance, the Cholesky factorization and algorithm cannot be performed [30]. In addition, the matrix representing the uncertainty of the sensor information cannot be resolved by Cholesky when the non-diagonal components are given a large value. Therefore, the experiment in each method gave the optimum value of the non-diagonal components of the error covariance matrix to 0.0001. Thus, in the second experiment, it has been confirmed that the estimated error reduced when the non-diagonal components of the error covariance matrix have been tested with zero. So, the chosen algorithm has been tested to ensure that the functions work correctly with the modified Cholesky factorization.

\section{Conclusions and future work}

In this research, the characteristics of the UKF method have been used to test the indoor mobile robot's location and compare and analyze its performance. The proposed method improved the estimation performance by presenting a new modeling method for the uncertainty value of the measurement sensor. The uncertainty value proportional to the measurement distance is designed to suit the actual environment better. Furthermore, it improves the estimation performance by setting the non-diagonal component values of the error covariance matrix to non-zero values in UT and Cholesky decomposition. The nondiagonal component was considered a non-zero value because the linear speed and rotational speed information used for estimation and sensor measurement distances influence each other. The UKF method needs to find good design parameter values to reduce the error range for the estimated position. The improved estimation results have been obtained between $2.2780<\gamma<2.775$ by changing the control parameters. When the control parameter is set large, the sigma point deviates from the boundary line, and the actual distance and the sensor distance calculated by the algorithm differ significantly. If the dispersion degree of the sigma point is small by setting the parameter small, it is corrected from the incorrectly predicted position to the original robot position. Hence, a new modeling method for the uncertainty of the measurement sensor has been proposed. Still, it is considered that future works on the uncertainty of the sensor information and the operation method for recognizing the situation are needed in an uncertain environment. 


\section{References}

[1] C Moon, JS Han and YA Kwon. Square-root unscented Kalman filter for state estimation of permanent magnet synchronous motor. In: Proceedings of the $55^{\text {th }}$ Annual Conference of the Society of Instrument and Control Engineers of Japan, Tsukuba, Japan. 2016, p. 460-4.

[2] S Holmes, G Klein and DW Murray. A square root unscented Kalman filter for visual monoSLAM. In: Proceedings of the 2008 IEEE International Conference on Robotics and Automation, Pasadena CA, United States. 2008, p. 3710-6.

[3] A Tsiami, A Katsamanis, P Maragos and G Potamianos. Experiments in acoustic source localization using sparse arrays in adverse indoors environments. In: Proceedings of the $22^{\text {nd }}$ European Signal Processing Conference, Lisbon, Portugal. 2014, p. 2390-4.

[4] T Fiorenzani, C Manes, G Oriolo and P Peliti. Comparative study of unscented Kalman filter and extended Kalman filter for position/attitude estimation in unmanned aerial vehicles. Istituto Di Analisi Dei Sistemi Ed Informatica, Roma, Italy, 2008, p. 1-30.

[5] Y Liu, Z Yang, X Wang and L Jian. Location, localization, and localizability. J. Comput. Sci. Tech. 2010; 25, 274-97.

[6] H Min, H Chen and R Luo. Active particle in MCL: An evolutionary view. In: Proceedings of the 2009 International Conference on Information and Automation, Zhuhai/Macau, China. 2009, p. 1087-92.

[7] K Ogata, H Tanaka and Y Matsumoto. A robust position and posture measurement system using visual markers and an inertia measurement unit. In: Proceedings of the 2019 IEEE/RSJ International Conference on Intelligent Robots and Systems, Macau, China, 2019, p. 7497-502.

[8] Z Dong, W Li and Y Zhou. An autonomous navigation scheme for UAV in approach phase. In: Proceedings of the 2016 IEEE Chinese Guidance, Navigation and Control Conference, Nanjing, China. 2016, p. 982-7.

[9] H Hlavacs and KA Hummel. Cooperative positioning when using local position information: Theoretical framework and error analysis. IEEE Trans. Mobile Comput. 2013; 12, 2091-104.

[10] M Rhudy, Y Gu, J Gross and M Napolitano. Sensitivity analysis of EKF and UKF in GPS/INS Sensor Fusion. In: Proceedings of the AIAA Guidance, Navigation, and Control Conference, Portland OR, United States. 2011.

[11] X Wang and Z Deng. Multi-model robust weighted fusion steady-state Kalman estimators with uncertain-covariance linearly correlated white noises. In: Proceedings of the $29^{\text {th }}$ Chinese Control and Decision Conference, Chongqing, China. 2017, p. 4873-9.

[12] S Shioda and K Shimamura. Relative localization of sensors based on their responses to moving objects. In: Proceedings of the IEEE $10^{\text {th }}$ International Conference on Mobile Ad-Hoc and Sensor Systems, Hangzhou, China. 2013, p. 419-20.

[13] F Martinelli. Robot localization: Comparable performance of EKF and UKF in some interesting indoor settings. In: Proceedings of the $16^{\text {th }}$ Mediterranean Conference on Control and Automation, Ajaccio, France. 2008, p. 499-504.

[14] Y Cheng and Z Liu. Optimized selection of sigma points in the unscented Kalman filter. In: Proceedings of the 2011 International Conference on Electrical and Control Engineering, Yichang, China. 2011, p. 3073-5.

[15] M Shyamalagowri and R Rajeswari. Unscented Kalman filter based nonlinear state estimation case study - nonlinear process control reactor (continuous stirred tank reactor). In: Proceedings of the $10^{\text {th }}$ International Conference on Intelligent Systems and Control, Coimbatore, India. 2016, p. 1-6.

[16] SR Malikov and J Ohlmann-Lauber. A contribution to the application of the $2 D$ laserscanner SICK LMS511 for deformation analysis. Institute of Geodesy, GIS and Land Management, München, Germany, 2014, p. 1-18.

[17] D Lee. Nonlinear estimation and multiple sensor fusion using unscented information filtering. IEEE Signal Process. Lett. 2008; 15, 861-4.

[18] OS Chernikova. An adaptive unscented Kalman filter approach for state estimation of nonlinear continuous-discrete system. In: Proceedings of the 2018 XIV International Scientific-Technical Conference on Actual Problems of Electronics Instrument Engineering, Novosibirsk, Russia. 2018, p. 37-40.

[19] M Cui, W Liu, H Liu, H Jiang and Z Wang. Extended state observer-based adaptive sliding mode control of differential-driving mobile robot with uncertainties. Nonlinear Dynam. 2015; 83, 667-63.

[20] D Li, Q Chen and Z Zeng. Self-localization algorithm of mobile robot based on unscented particle filter. In: Proceedings of the $37^{\text {th }}$ Chinese Control Conference, Wuhan, China. 2018, p. 5459-64. 
[21] LE Padilla and CW Rowley. An adaptive-covariance-rank algorithm for the unscented Kalman filter. In: Proceedings of the $49^{\text {th }}$ IEEE Conference on Decision and Control, Atlanta GA, United States. 2010, p. 1324-9.

[22] Z Tang, C Sun and Z Liu. The tracking algorithm for maneuvering target based on adaptive Kalman filter. Int. Arab J. Inform. Tech. 2013; 10, 453-9.

[23] S Han, W Wang, X Chen, W Meng. Design and capability analyze of high dynamic carrier tracking loop based on UKF. In: Proceedings of the $23^{\text {rd }}$ International Technical Meeting of the Satellite Division of the Institute of Navigation, Portland OR, United States. 2010.

[24] R Aucoin, SA Chee and JR Forbes. Linear- and linear-matrix-inequality-constrained state estimation for nonlinear systems. IEEE Trans. Aero. Electron. Syst. 2019; 55, 3153-67.

[25] L Li and Y Xia. Stochastic stability of the unscented Kalman filter with intermittent observations. Automatica 2012; 48, 978-81.

[26] S Tomita, K Sekiyama and T Fukuda. Consensus making algorithms based on invariants perception for cognitive sharing in multi-robot. In: Proceedings of the $2^{\text {nd }}$ International Conference on Robot, Vision and Signal Processing, Kitakyushu, Japan. 2013, p. 220-3.

[27] AA Arabi, P Sarkar, F Ahmed, WR Rafie, M Hannan and MA Amin. 2D mapping and vertex finding method for path planning in autonomous obstacle avoidance robotic system. In: Proceedings of the $2^{\text {nd }}$ International Conference on Control and Robotics Engineering, Bangkok, Thailand. 2017, p. 39-42.

[28] W Yuhuan, W Jinkuan and W Bin. A modified multi-target tracking algorithm based on joint probability data association and Gaussian particle filter. In: Proceedings of the $11^{\text {th }}$ World Congress on Intelligent Control and Automation, Shenyang, China. 2014, p. 2500-4.

[29] T Schlegl, T Bretterklieber, M Neumayer and H Zangl. A novel sensor fusion concept for distance measurement in automotive applications. In: Proceedings of the 2010 IEEE Sensors, Kona HI, United States. 2010, p. 775-8.

[30] A Krishnamoorthy and D Menon. Matrix inversion using Cholesky decomposition. In: Proceedings of the 2013 Signal Processing: Algorithms, Architectures, Arrangements, and Applications, Poznan, Poland. 2013, p. 70-2.

[31] JM Li, CW Chen and TH Cheng. Estimation and tracking of a moving target by unmanned aerial vehicles. In: Proceedings of the American Control Conference, Philadelphia PA, United States. 2019. p. 3944-9.

[32] S Erfani, A Jafari, A Hajiahmad. Comparison of two data fusion methods for localization of wheeled mobile robot in farm conditions. Artif. Intell. Agr. 2019; 1, 48-55.

[33] D Nada, M Bousbia-Salah and M Bettayeb. Multi-sensor data fusion for wheelchair position estimation with unscented Kalman Filter. Int. J. Autom. Comput. 2018, 15, 207-17. 